\title{
Role of response time of a Babcock-Leighton solar dynamo model in meridional flow-speed reconstruction by EnKF data assimilation
}

\author{
Mausumi Dikpati \\ High Altitude Observatory, National Center for Atmospheric Research, \\ 3080 Center Green Dr., Boulder, CO 80301, USA \\ email:dikpati@ucar.edu \\ Dhrubaditya Mitra \\ NORDITA, Roslagstullsbacken 2310691 Stockholm, \\ Sweden \\ Jeffrey L. Anderson \\ IMAGe, National Center for Atmospheric Research, \\ 1850 Table Mesa Drive, Boulder, CO 80305, USA
}

\begin{abstract}
Ensemble Kalman Filter in the framework of Data Assimilation Research Testbed (DART) has been successfully implemented into a 2D kinematic flux-transport dynamo model by Dikpati and colleagues in order to do a parameter estimation, the parameter being the meridional flow-speed as function of time. They performed several 'Observing System Simulation Experiments' (OSSEs), and showed that an optimal reconstruction of time-series of meridional flow-speed can be obtained by using 16 ensemble members and only one surface magnetic observation with $30 \%$ observational error. Error in reconstruction can be reduced by increasing the ensemble size and number of observations. However, this parameter reconstruction has been found to be sensitive to locations from where observational data are taken. While assimilation of low-latitudes' surface poloidal magnetic field data can produce good reconstruction, medium-frequency oscillations appear in time-series of reconstructed flow-speed if tachocline toroidal field data are assimilated. These oscillations occur primarily because tachocline toroidal fields change very little during an assimilation interval taken to be 15 days, due to changes in meridional flow. A Babcock-Leighton dynamo model's response time to changes in meridional flow-speed is a few months. We show here that rms error in reconstruction can be significantly reduced if model's response time is taken into consideration in assimilation of tachocline toroidal field data.
\end{abstract}

Preprint submitted to Elsevier Science

2 August 2016

(C) 2016. This manuscript version is made available under the Elsevier user license http:/www.elsevier.com/open-access/userlicense/1.0/ 
Key words: Sun: magnetic fields, meridional circulation, dynamo model, data assimilation

\section{INTRODUCTION}

Babcock-Leighton type flux-transport solar dynamo models in both 2D (Wang \& Sheeley , 1991; Choudhuri, Schüssler \& Dikpati , 1995; Dikpati \& Charbonneau , 1999; Guerrero, Dikpati \& de Gouveia Dal Pino , 2009; Nandy, Muñoz-Jaramillo \& Martens , 2011; Belucz, Dikpati \& Forgács-Dajka , 2015) and 3D (Yeates \& Muñoz-Jaramillo , 2013; Miesch \& Dikpati , 2014) have been successful in explaining many large-scale solar cycle features, including a particularly difficult one - the correct phase relationship between the equatorward migrating sunspot belt and the poleward drifting large-scale, diffuse fields. This class of models have four major dynamo ingredients, namely the differential rotation, meridional circulation, Babcock-Leighton type surface poloidal source and magnetic diffusivity. These models can be best calibrated for the Sun (see, e.g. Dikpati et al (2004)) if they operate with a primarily single-celled meridional circulation pattern in each hemisphere.

The meridional circulation, along with magnetic diffusivity, play crucial roles in determining (i) the dynamo cycle period and (ii) governing the memory of the model's past magnetic fields. However, the profile and spatio-temporal pattern of this circulation is known at/near the surface down to a shallow layer, not in the deeper convection zone and tachocline. Even in the upper half of the convection zone, different observations infer different profiles. Using timedistance helioseismology the most recent observations from SDO/HMI data infer meridional circulation with two cells in depth (Zhao et al. , 2013). Ring-diagram analysis from GONG data gives poleward surface flow up to about $\sim 60^{\circ}$ latitude (Komm et al. , 2013), whereas Doppler measurements from MWO data, which can measure this flow at higher latitudes, show a high-latitude, reverse flow associated with the primary poleward surface flow (Ulrich , 2010). Using a very long-term GONG database and applying timedistance technique Kholikov, Serebryanskiy \& Jackiewicz (2014) have found signatures of equatorward return-flow in the lower half of the convection zone, indicating a long deep one cell flow-pattern. A p-mode perturbation analysis by Schad, Timmer \& Roth (2013) yields four cells in latitude, each going down to about0.8R. Thus observations do not yet give us a unique answer about the Sun's meridional circulation pattern.

Models produce more complex flow patterns that vary from model to model. For example, mean-field models produce a long, counterclockwise primary cell often associated with a weak, reverse cell at high latitudes, both extending down to the bottom of convection zone (Dikpati , 2014), whereas full 3D 
convection simulations produce multiple cells in latitude and depth (Guerrero et al , 2013; Featherstone \& Miesch , 2015).

Recently Dikpati, Anderson \& Mitra (2014) have demonstrated that Ensemble Kalman Filter data assimilation method in the DART framework has the potential for successfully reconstructing the time-variation of the Sun's meridional circulation. By building a Dynamo-DART system, Dikpati et al. (2014) performed Observing System Simulation Experiments (OSSEs) using a 2D Babcock-Leighton flux-transport dynamo model operating with a timevarying meridional flow-speed, which they assumed. Note that in OSSEs, the observational data are "synthetic" data, which are obtained by adding noise to model-output. Dikpati et al. (2014) found that their Dynamo-DART system can best reconstruct the time variation in meridional flow-speed if 160 ensemble members are used in assimilation of 180 magnetic field observations with $1 \%$ observational error in each of them, although a fairly reasonable reconstruction of meridional flow-speed was possible by just using only 16 ensemble members and one observation with $30 \%$ error.

By performing several OSSEs, these authors showed recently (Dikpati, Anderson \& Mitra , 2016) that the reconstruction is sensitive to locations from where observational data is taken. For example, a very good reconstruction of the time series of meridional flow-speed is obtained when surface poloidal magnetic field observations from low latitudes are assimilated, but the reconstructed meridional flow-speed shows some oscillations when tachocline toroidal field data are assimilated.

This medium-frequency oscillation occurs due to the fact that the assimilation interval of 15 days is too short a time for the tachocline toroidal fields to respond to the changes in meridional circulation. A Babcock-Leighton solar dynamo model's overall response time to changes in meridional circulation is a few months (see, e.g. Dikpati \& Anderson 2012). Here we show that such oscillations in the time series of meridional flow-speed can be reduced if the information of the model's response time is given to the Dynamo-DART system.

In the next section we describe the methodology for including the dynamo model's response time in the assimilation. We present our results in $\S 3$ and close the paper with concluding remarks.

\section{DESCRIPTION OF METHODOLOGY}

The Data Assimilation Research Testbed (DART) is a flexible sequential data assimilation framework that allows experimenting with different filter 
algorithms, such as Ensemble Kalman Filter (EnKF), Ensemble Adjustment Kalman Filter (EAKF) conveniently. More details on the method can be found in Anderson (2001) and Anderson \& Collins (2007), and the software is available in IMAGe department of NCAR/CISL (Computer Information System Laboratory) from the url http://www.image.ucar.edu/DAReS/DART. The EnKF-DART scheme is based on statistical multidimensional regression analysis using a Bayesian approach - a well-developed method that has been applied in atmospheric and oceanic studies, such as in the Earth's upper atmosphere models (see Matsuo et al. 2013), and recently in a 2D Babcock-Leighton solar dynamo model (Dikpati et al. 2014).

A different EnKF scheme was applied in the past (Kitiashvili \& Kosovichev , 2008) in a one-dimensional solar dynamo model for predicting solar cycle 24 amplitude. For real-life predictions of polar field patterns, ADAPT scheme based on EnKF data assimilation has been implemented by Arge et al (2010) and Hickmann, Godinez, Henney \& Arge (2015). Fournier, Nerger \& Aubert (2013) have implemented the EnKF data assimilation scheme in a geodynamo model.

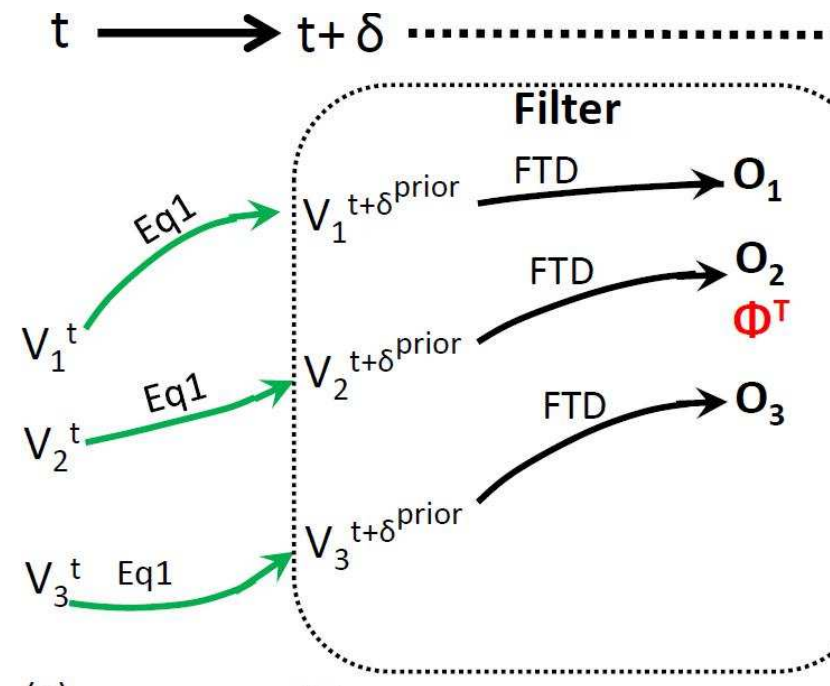

(a)

(b)

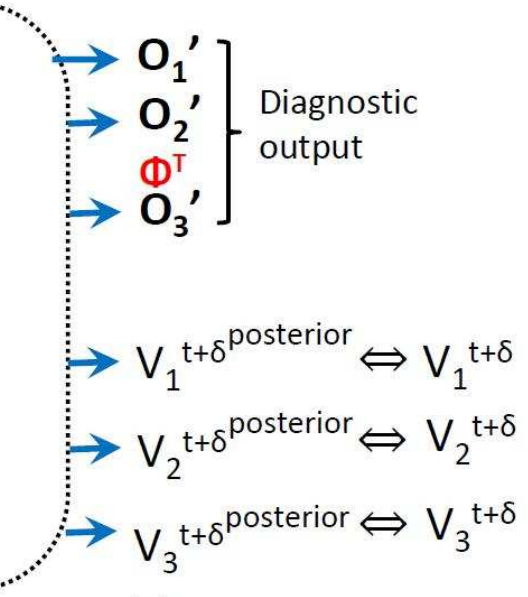

(c)

Fig. 1. Schematic representation of the EnKF method as applied in present problem. Three members of the ensemble of flow-speeds at time $t$, namely $V_{1}^{t}, V_{2}^{t}$ and $V_{3}^{t}$ are evolved using Equation (1) to generate corresponding prior state at time $t+\delta$, shown as evolution from (a) to (b). In the "Filter" box, observations $O_{1}, O_{2}$ and $\mathrm{O}_{3}$ are generated by applying forward operator (FTD) on the prior estimates. Then following a linear regression analysis on these observations with corresponding flow-speeds, the "Filter" estimates posterior flow-speeds using the synthetic observation with errors. In the process it also generates the posterior observation for evaluation of innovation at time $t+\delta$. Time advances from $t$ to $t+\delta$ (denoted by solid line at the top) during the evolution from (a) to (b), while in the "Filter" procedure, time remains frozen at step $t+\delta$ (denoted by dotted line from (b) to (c)) (adopted from Dikpati, Anderson \& Mitra (2014)). 
Following Dikpati, Anderson \& Mitra (2014), we will again implement here the EnKF data assimilation in a Babcock-Leighton flux-transport (BLFT) dynamo model of Dikpati et al (2010). Note that the physical model (i.e. BLFT dynamo model) is the forward operator in data assimilation terminology, and we call it FTD. Our goal is to investigate the role of the dynamo model's response time to changes in meridional flow in reconstructing the time variation in meridional flow-speed. The time-varying meridional flow-speed at a given time is the "model state" or "true state", to be estimated by the EnKF system. For convenience of comparison between the true state of the time-varying meridional flow-speed and the flow-speed reconstructed from ensemble mean, we present the true state in Figure 2, which is adopted from figure 2(a) of Dikpati, Anderson \& Mitra (2014).

EnKF sequential data assimilation is a two-step procedure, a forecast followed by an analysis each time an observation is available. In the first step, from initial state vectors at time $t$, a forecast at time $t+\delta$ is made using an assimilation model (the BLFT dynamo model here); thus the "prior states" of the state vectors are generated. In the second step of the two-step process, their "posterior states" are estimated by employing multidimensional regression among the observation vectors and the prior state vectors. Figure 1 schematically describes these steps (see Dikpati, Anderson \& Mitra (2014) for details).

In Figure $1, v_{1}^{t}, v_{2}^{t}, v_{3}^{t}$ near the label (a) denote three different realizations of initial flow-speeds. The EnKF requires a prediction model, given by Equation (1), that generates a forecast of the meridional flow-speed at a later time given the value at the current time (i.e., $\left.v_{1}{ }^{t}, v_{2}{ }^{t}, v_{3}{ }^{t}\right)$ :

$$
v_{1}^{t+\delta^{\text {prior }}}=v_{1}^{t}+\Gamma_{0} \times \gamma
$$

in which, $\gamma$ is a function for generating normalized Gaussian random numbers with unit amplitude and unit standard deviation, and the amplitude of the prediction model noise is governed by $\Gamma_{0}$. "Prior states", denoted by $v_{1}{ }^{t+\delta^{\text {prior }}}, v_{2}{ }^{t+\delta^{\text {prior }}}, v_{3}{ }^{t+\delta^{\text {prior }}}$ near the label $\left.(\mathrm{b})\right)$ are generated by using Equation (1) to advance the estimates of the flow-speed from the initial time $(t)$ to the time $(t+\delta)$ at which the first observations of magnetic field are available.

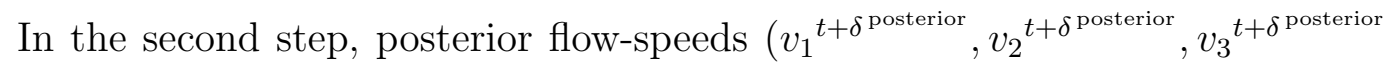
are estimated by employing regression among the model-outputs of magnetic fields, "synthetic" observations of magnetic fields and prior flow-speeds. flowspeeds. The forward operator (BLFT dynamo in this case) is denoted by FTD along the solid black arrows, which uses the prior estimates of flow speed to produce a prior estimates of ensemble of magnetic field observations. Three realizations of magnetic fields from the forward operator are denoted by $O_{1}$, $\mathrm{O}_{2}, \mathrm{O}_{3}$ in Figure 1.

Medium frequency oscillations in the reconstruction occur because changes in 
tachocline magnetic fields during 15 days of assimilation interval are generally small due to changes in flow speed. The flow-speed is much smaller at/near the base of the convection zone than at the surface because of the density stratification. So a plausible remedy can be to run the model longer than the assimilation interval of 15 days, and obtain significant changes in the tachocline magnetic fields. So we again consider an assimilation interval of 15 days, but another longer time span (say 30 days) during which the flow speed continues to vary with time so that significant changes in tachocline magnetic fields occur. So we start integration of the forward operator at time $t$ and continue the integration up to $t+30$ days; we obtain the model-outputs at the time $t+30$ days, and consider the "synthetic" observations also at $t+30$ days, and regress them with prior states of flow-speeds. The posterior states of velocities obtained from this regression we implement as prior states at the time of $t+15$ days instead of at the time $t+30$ days. In this way, we keep our assimilation interval as 15 days, but apply an operation time ( $>15$ days) to the forward operator for using its response time.

\section{RESULTS}

We consider 10 toroidal magnetic field observations, which are taken from the center of the tachocline, particularly from the depth of $0.704 R$, and from the low latitudes ranging from $23.4^{\circ}$ to $31.5^{\circ}$ with an interval of $0.9^{\circ}$ magnetic field observations, and perform assimilation runs with 192 ensemble members with an observational error of $\sim 30 \%$, which means an error of $\sim 30 \%$ about the ideal magnetic field generated using the true meridional flow speed. To estimate the prior states of flow-speed, we use Equation (1), in which we set $\Gamma_{0}=0.5 \mathrm{~ms}^{-1}$. If the meridional flow-speed varies up to $\pm 20 \%$ (i.e. $\sim \pm 2.8 \mathrm{~ms}^{-1}$ for a mean flow-speed of $14 \mathrm{~ms}^{-1}$ ) during six months, the variation in 15 days can be $\sim 0.23 \mathrm{~ms}^{-1}$. Thus we chose $\Gamma_{0}=0.5 \mathrm{~ms}^{-1}$ so that it is large enough to capture the variation in flow-speed within our selected updating time-step of 15 days and also large enough to avoid ensemble collapse, but not so large as to produce unusual departures from cyclic behavior in a flux-transport dynamo.

We present the results in three panels of Figure 2; panel (a) shows the true state of the time-varying flow-speed to be reconstructed by EnKF assimilation in our DART-Dynamo system. The reconstructed flow-speed, obtained by assimilating 10 synthetic observations of low-latitude tachocline magnetic fields in an assimilation interval of 15 days, has been plotted in panel (b). 


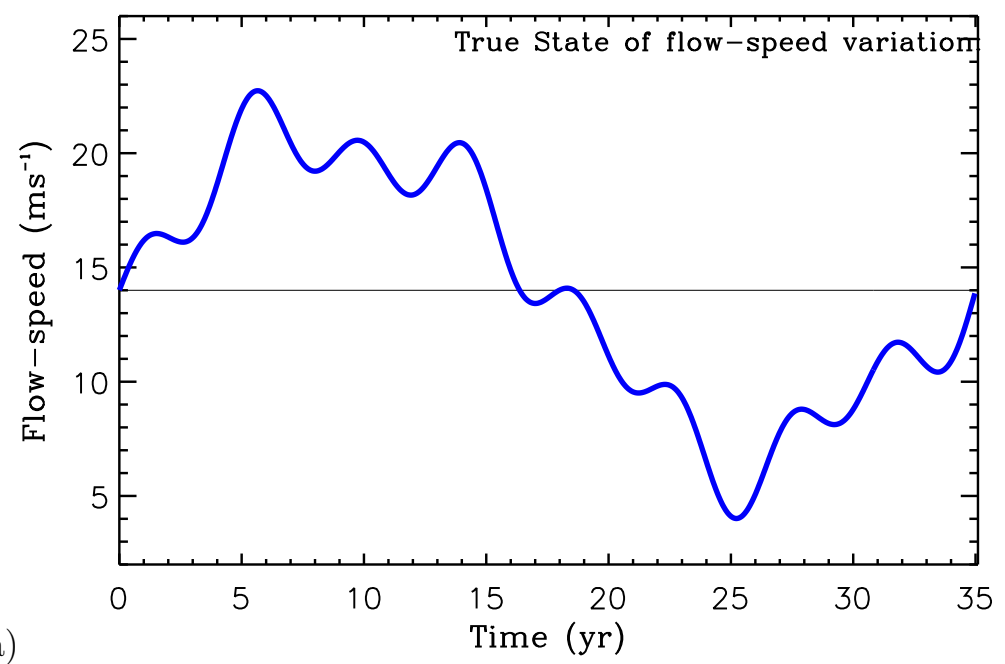

(a)

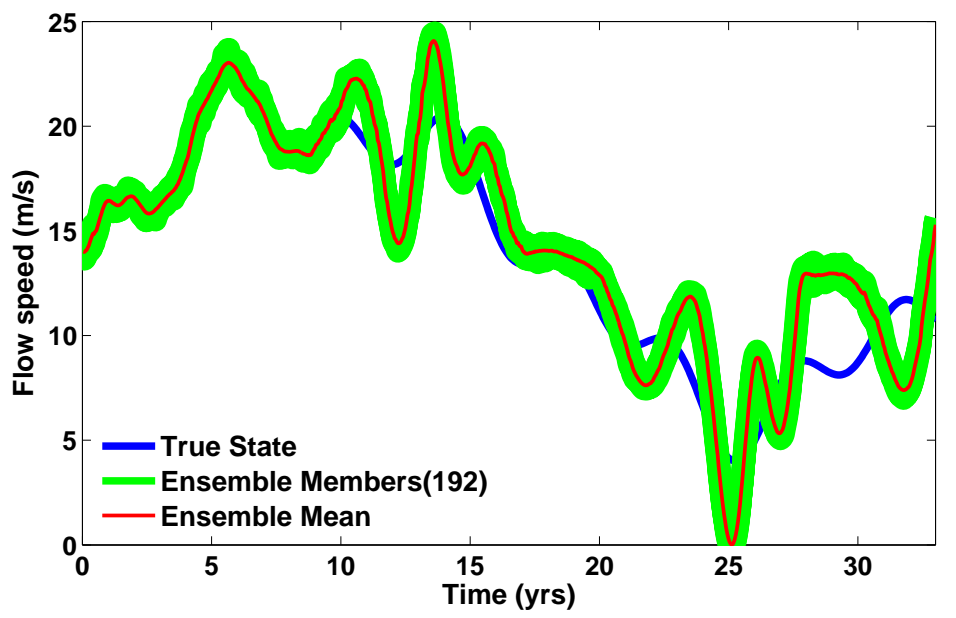

(b)

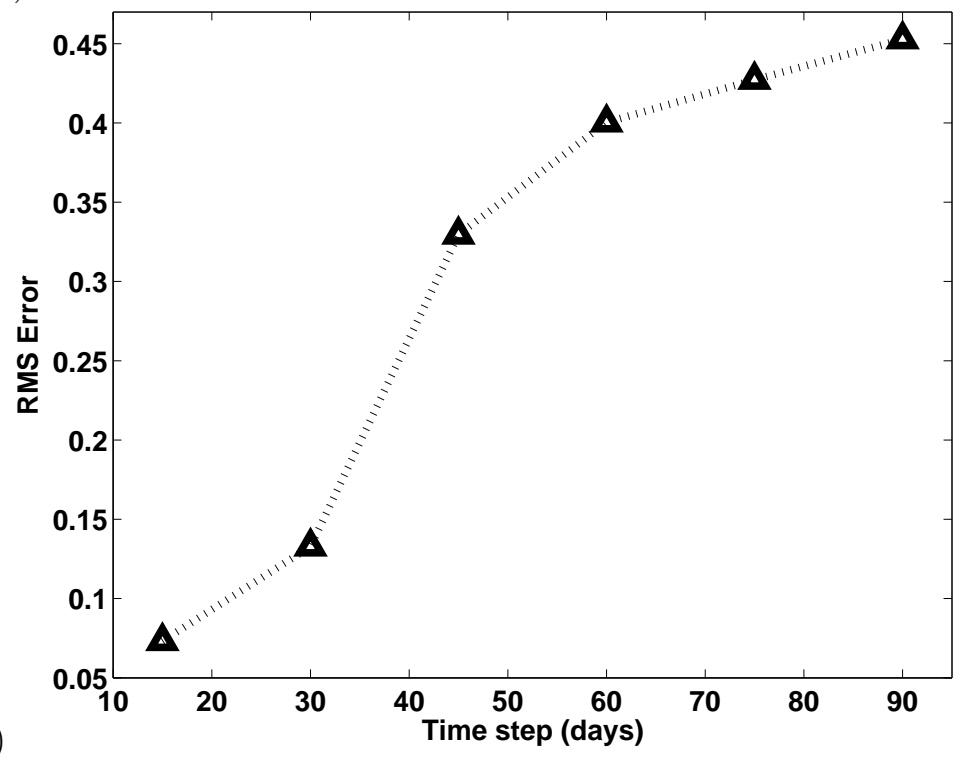

Fig. 2. Panel (a): true state (i.e. time-varying meridional flow speed) that varies by $\pm 70 \%$ about mean flow-speed, $14 \mathrm{~ms}^{-1}$; Panel (b): red curve denotes ensemble-mean of reconstructed meridional flow-speeds as a function of time; superimposed green curves denote all 192 ensemble members; true state is plotted in blue for comparison. Panel (c): rms error in reconstruction as function of assimilation time interval. 
Defining the rms error in reconstruction as, $\sqrt{\frac{\sum_{i=1}^{n_{\max }}\left(v_{T}{ }^{i}-v_{R}\right)^{2}}{n_{\max }}}$, in which $i$ denotes the index for every assimilation interval, $v_{T}{ }^{i}$ the true state at the $i$ th assimilation step and $v_{R}{ }^{i}$ the reconstructed state, and $n_{\max }$ the total number of assimilation steps during the entire time span of the assimilation runs, we plot the rms error as function of assimilation interval and present in panel (c). Panel (b) of Figure 2 reveals that the reconstructed flow-speed starts deviating from the truth after 10 years after which medium frequency oscillations in the reconstructed flow speed occur, when the tachocline magnetic fields are used. Apart from oscillations, we find that the reconstruction is showing major deviations with opposite trends with respect to the true state late in the assimilation runs, namely beyond $t=27$ years.

Variation in tachocline toroidal magnetic fields is much smaller than that in surface magnetic fields, because (i) the flow-speed is smaller at the bottom of the convection zone and in the tachocline, (ii) the magnetic diffusivity is smaller there than in the upper half of the convection zone and at the surface. So the flux-freezing inertia felt by the tachocline toroidal magnetic fields is large, and these fields are not prone to significant changes in 15 days by flowspeed variation. As a consequence the reconstructed flow-speed deviates from the true state. If we just assimilate only one observation of tachocline toroidal field, the reconstruction continues to deviate without approaching towards the truth. However the assimilation of more than one observations of tachocline toroidal fields produces such deviations at different times, and the net result is a back and forth oscillation in reconstruction about the truth.

The obvious question arises whether the reconstructions can be improved if the assimilation interval is increased. To answer this question, we run the assimilations using different assimilation time intervals, and plot in Figure 2(c) the rms error in reconstruction as function of assimilation time interval. Figure 2 (c) reveals that increasing the assimilation interval is not the answer, because the reconstruction deteriorates with longer updating intervals. The optimum assimilation interval is about 15 days. Reconstruction starts deteriorating again when assimilation interval is decreased from 15 days to a smaller value. By performing extensive assimilation runs, Dikpati, Anderson \& Mitra (2016) have recently shown in their figure 14 that the RMS error in reconstruction is indeed minimum for 15 days assimilation interval. 


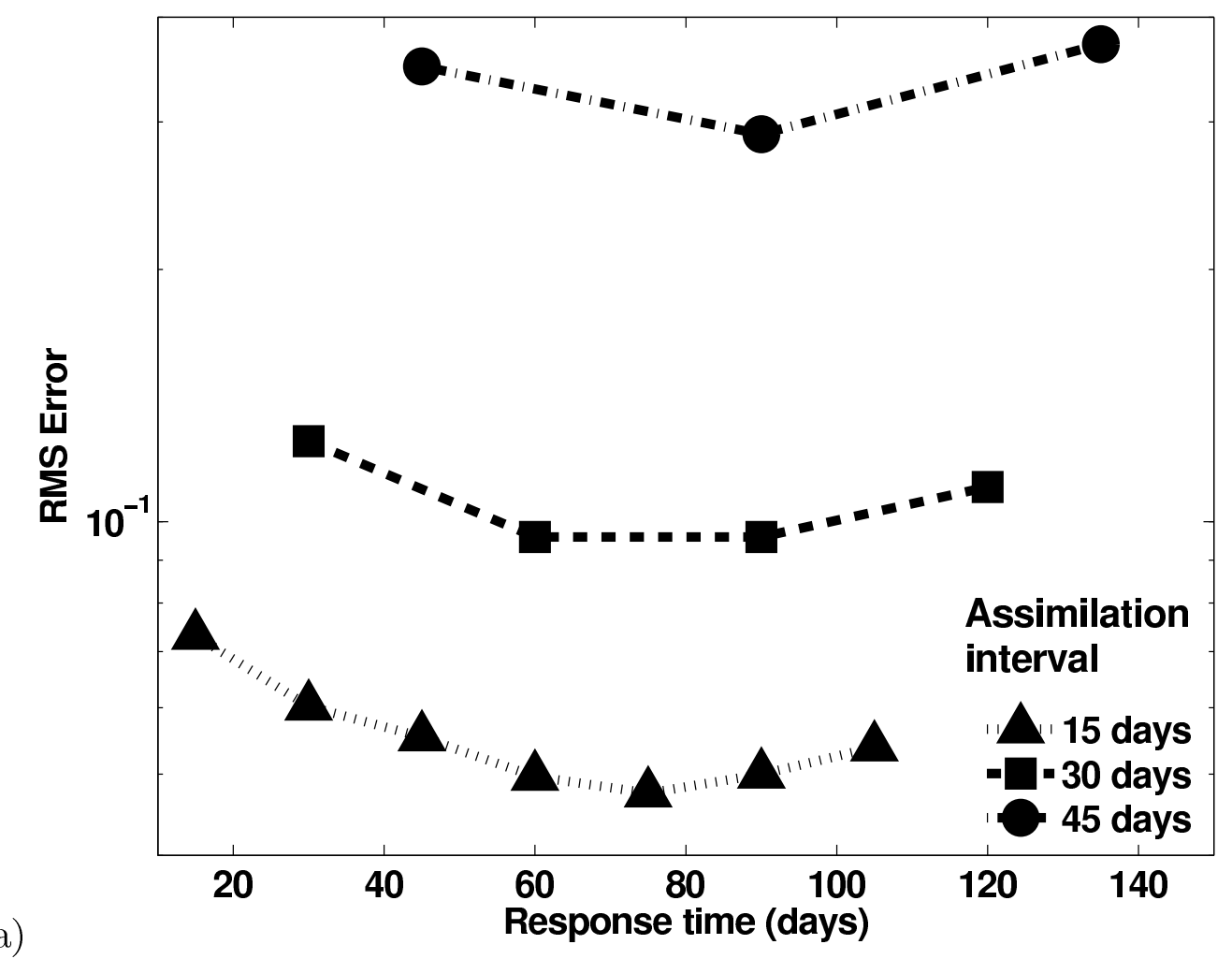

(a)

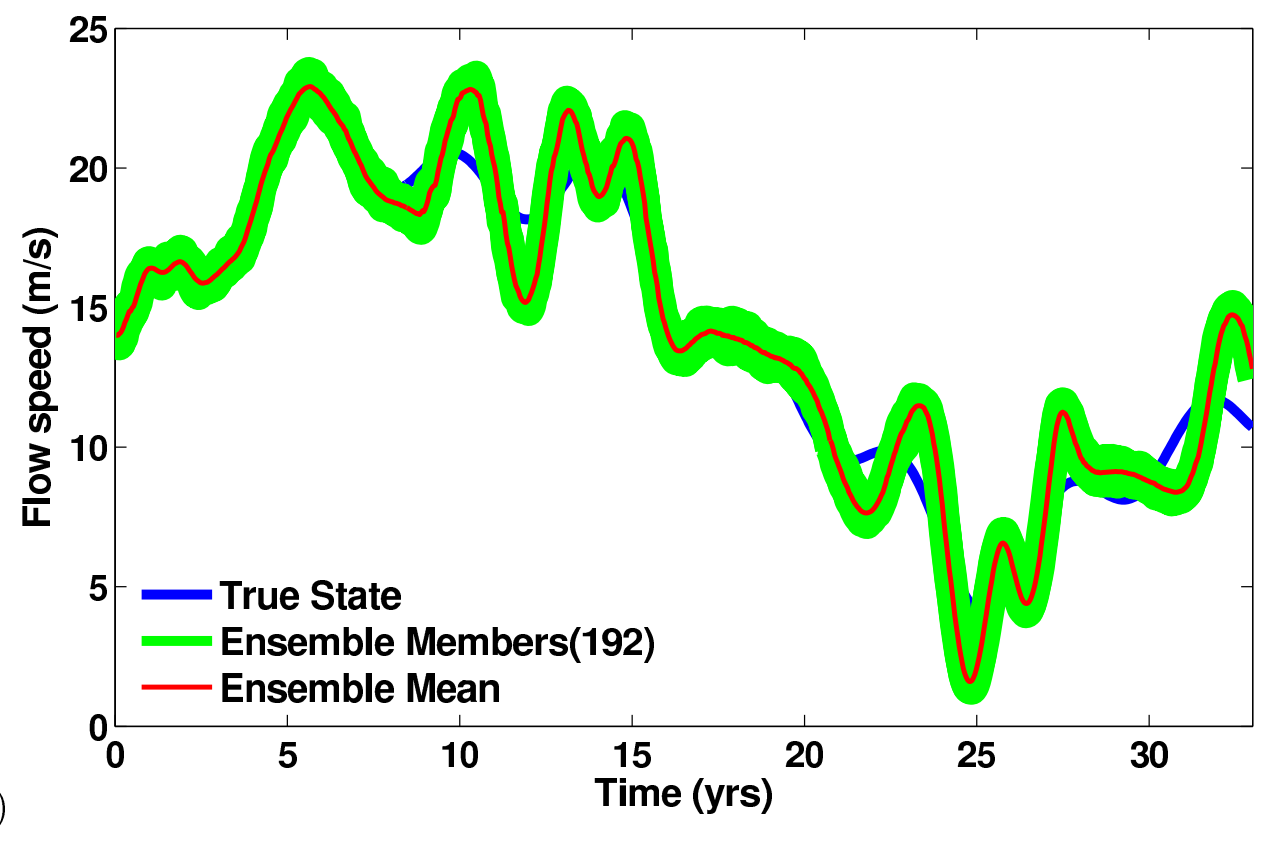

Fig. 3. (a) RMS errors in reconstruction (in units of $\mathrm{ms}^{-1}$ ) as functions of response time for assimilation intervals of 15,30 and 45 days, respectively plotted in solid triangles, squares and circles implemented in the assimilation runs; (b) reconstructed flow-speed as function of time, obtained by implementing 75 days response time. 
Speculating that allowing a response time of a few months, or in other words, allowing the model to feel the change of meridional flow speed for a few months, can improve the reconstruction, we perform the assimilation runs using different response times, using three different assimilation intervals - 15, 30 and 45 days. We present our results in Figure 3; the upper panel shows the rms error in reconstruction as function of response time, for assimilation intervals of 15, 30 and 45 days, respectively plotted in solid triangles, squares and circles. The lower panel shows the reconstructed flow-speed for the case with 75 days response time. We find in Figure 3(a) that the, for each assimilation interval chosen, the rms error in reconstruction decreases with the increase in response time up to a certain value (75, 90 and 90 days respectively), and then starts increasing again. Note that the response time can be only the integral multiple of assimilation interval in these experiments. Examining the three curves in Figure 3(a) we can clearly find that the minimum RMS error in reconstruction can be obtained when 15 days assimilation interval is used. For a Babcock-Leighton dynamo model, updating the Dynamo-DART system in every 15 days is optimal (see figure 14 of Dikpati, Anderson \& Mitra (2016); see also Sanchez, Fournier \& Auburt (2014), for discussion on optimal updating interval in a Babcock-Leighton dynamo model). Therefore, a corresponding curve of RMS error as function of response time for 5 days assimilation interval will shift upward of solid triangles.

Figure 3(b) shows that the reconstructed flow-speed is largely improved compared to that in Figure 2(b); the oscillations in reconstructions are much reduced, but they are not completely gone. The opposite trends in the time window of 27 to 35 years are rectified. However, this reconstruction is still poorer than the best reconstruction obtained by assimilating surface magnetic observations, as was seen in figure 5 of Dikpati, Anderson \& Mitra (2014).

Dikpati, Anderson \& Mitra (2014) used 180 observations from the surface and near surface region. We perform here an experiment to examine how much improvement we can obtain if we include one surface poloidal field observation and nine tachocline toroidal field observations. Nine tachocline toroidal field observations are taken from the tachocline center and from the latitude range of $23.4^{\circ}-30.6^{\circ}$, and the surface poloidal field observation is taken from $0.98 R$ and $4.5^{\circ}$ latitude. We present the result in Figure 4 . We can see that the reconstruction is greatly improved when we include surface magnetic observations along with tachocline magnetic field observations.

The reasons for this significant improvement in reconstruction by including surface poloidal field observation in the assimilation runs is that the flow speed at the surface is about 10 to 15 times larger than that at the bottom of the convection zone; therefore the variation in flow speed is also larger there. Furthermore, the magnetic diffusivity being larger near the surface, 


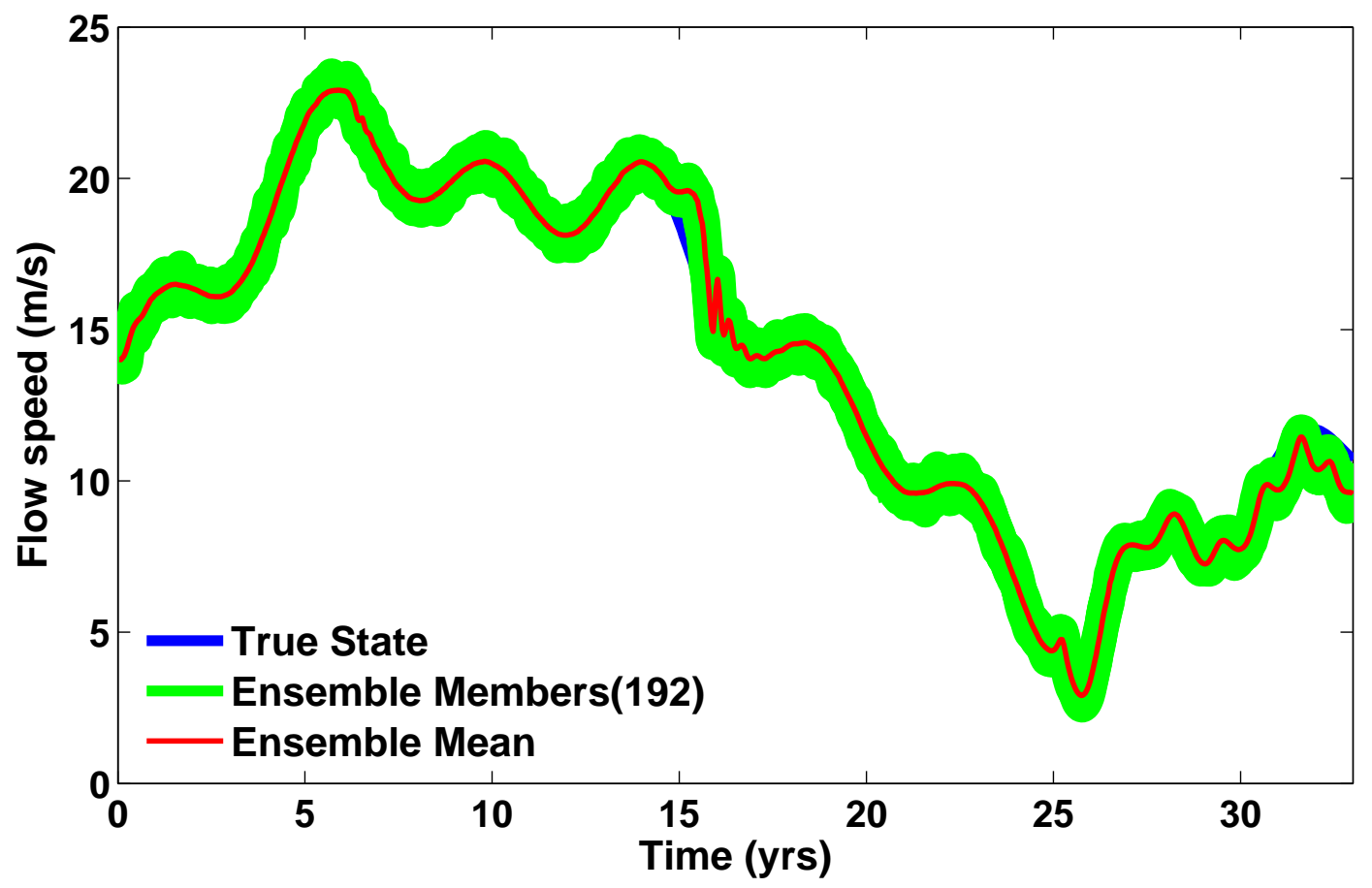

Fig. 4. Same as in Figure 2(b) but with incorporation of response time in the assimilation runs using nine tachocline toroidal magnetic field and one surface poloidal field observations.

the flux-freezing inertia is much less for the surface poloidal fields. Thus the changes in the poloidal fields in 15 days, due to variation in flow speed, is significant, but also not too large to be uncorrelated to the poloidal fields of the previous assimilation, i.e. the poloidal fields at that location 15 days ago. So the resulting reconstruction is largely improved.

\section{COMMENTS}

It was previously demonstrated through OSSEs that EnKF data assimilation into a DART-Dynamo system can be a powerful tool for successfully reconstructing time-variation in meridional circulation speed for more than a solar cycle from observations of the magnetic field. However, the reconstruction is sensitive to the locations from where observations are taken. Assimilation of tachocline magnetic field observations produce oscillations affecting the faithful reconstruction.

We demonstrated here that these oscillations can be reduced and reconstruction can be improved up to a certain extent if the physical model's response time is taken into consideration and OSSEs are performed with incorpora- 
tion of such response time. However, in order to obtain the most faithful reconstruction of time variation in meridional flow-speed it is necessary to include surface observations of weak poloidal magnetic fields along with strong tachocline magnetic field observations.

One of the major purposes of data assimilation techniques is to estimate parameters which are difficult to obtain from models and/or observations. Application of data assimilation technique is fairly new in solar physics, but the implementation of both the EnKF techniques and variational methods in solar models have been demonstrated to be successful in the reconstructions of different types of parameters, such as $\alpha$-effect (see, Jouve, Brun \& Talagrand (2011)), time series of meridional circulation (Dikpati, Anderson \& Mitra , 2014). These observing system simulation experiments are very important for eventually assimilating instruments' data in the model for real time predictions.

\section{Acknowledgments}

We thank Hanli Liu for reviewing the entire manuscript. We extend our thanks to two anonymous reviewers for their helpful comments, which have helped improve the paper significantly. Our thanks go to Nancy Collins and Tim Hoar for their invaluable help with assimilation tools and software. The DART/Dynamo assimilation runs have been performed on the Yellowstone Supercomputer of NWSC/NCAR under project number P22104000, and two NCAR Strategic Capability project with award numbers NHAO0009 and NHAO0010. All assimilation tools used in this work are available to the public from http : //www.image.ucar.edu/DAReS/DART. National Center for Atmospheric Research is sponsored by National Science Foundation.

\section{References}

Anderson, J. L., An ensemble adjustment Kalman filter for data assimilation, Mon. Wea. Rev., 129, 2884-2903, 2001

Anderson, J. \& Collins, N., J., Scalable Implementations of Ensemble Filter Algorithms for Data Assimilation, Journal of Atmos. and Oceanic Tech., 24, 1452-1463, 2007

Arge, C. N., Henney, C. J., Koller, J., Compeau, C. R., Young, S., MacKenzie, D., Fay, A. \& Harvey, J. W., Solar Wind 12: Proceedings of Twelfth International Solar Wind Conference 1216, AIP, Saint-Malo, France, 343-364, 2010 
Belucz, B., Dikpati, M. \& Forgács-Dajka, E., A Babcock-Leighton Solar Dynamo Model with Multi-cellular Meridional Circulation in Advection- and Diffusion-dominated Regimes, ApJ, 806, 169, 18pp, 2015

Choudhuri, A. R., Schüssler, M. \& Dikpati, M., The solar dynamo with meridional circulation, Astron. Astrophys., 303, L29-L32, 1995

Dikpati, M. \& Charbonneau, P., A Babcock-Leighton Flux Transport Dynamo with Solar-Like Differential Rotation, ApJ, 518, 508-520, 1999

Dikpati, M., de Toma, G., Gilman, P. A., Arge, C. N. \& White, O. R., Diagnostics of Polar Field Reversal in Solar Cycle 23 Using a Flux Transport Dynamo Model, ApJ, 601, 1136-1151, 2004

Dikpati, M., Gilman, P. A., de Toma, G., \& Ulrich, R. K., Impact of changes in the Sun's conveyor-belt on recent solar cycles, Geophys. Res. Lett., 37, L14107, 1-6, 2010

Dikpati, M. \& Anderson, J. L., Evaluating Potential for Data Assimilation in a Flux-transport Dynamo Model by Assessing Sensitivity and Response to Meridional Flow Variation, ApJ, 756, 20, 1-14, 2012

Dikpati, M., Anderson, J. L. \& Mitra, D., Ensemble Kalman filter data assimilation in a Babcock-Leighton solar dynamo model: An observation system simulation experiment for reconstructing meridional flow speed, Geophys. Res. Lett., 41, 15, 5361-5369, 2014

Dikpati, M., Generating the Sun's global meridional circulation from differential rotation and turbulent Reynolds stresses, MNRAS, 438, 2380-2394, 2014

Dikpati, M., Anderson, J. L. \& Mitra, D., Data assimilation in a solar dynamo model using Ensemble Kalman Filters: Sensitivity and robustness in reconstruction of meridional flow-speed, ApJ, (in press), 2016

Featherstone, N. \& Miesch, M. S., Meridional Circulation in Solar and Stellar Convection Zones, ApJ, 804, 67, 21pp, 2015

Fournier, A., Nerger, L. \& Aubert, J., An ensemble Kalman filter for the time-dependent analysis of the geomagnetic field, Geochemistry, Geophys, Geosystems, 14, 10, 4035-4043, 2013

Guerrero, G. A., Dikpati, M. \& de Gouveia Dal Pino, E. M., The Role of Diffusivity Quenching in Flux-transport Dynamo Models, ApJ, 705, 725736,2009

Guerrero, G., Smolarkiewicz, P. K., Kosovichev, A. G. \& Mansour, N. N., Differential Rotation in Solar-like Stars from Global Simulations ApJ, 779, 176, 13pp, 2013

Hickmann, K. S., Godinez, H. C., Henney, C. J \& Arge, C. N., Data Assimilation in the ADAPT Photospheric Flux Transport Model, Sol. Phys., 290, 1105-1118, 2015

Jouve, L., Brun, A. S. \& Talagrand, O., Assimilating Data into an $\alpha-\Omega$ Dynamo Model of the Sun: A Variational Approach, ApJ, 735, 31, 1-14, 2011

Kholikov, S., Serebryanskiy, A. \& Jackiewicz, J., Meridional Flow in the Solar Convection Zone. I. Measurements from GONG Data, ApJ, 784, 145, 1-5, 2014 
Kitiashvili, I. \& Kosovichev, A. G., Application of Data Assimilation Method for Predicting Solar Cycles, ApJ Lett., 688, L49-L52, 2008

Komm, R., González-Hernández, I., Hill, F., Bogart, R., Rabello-Soares, M. C. \& Haber, D., Subsurface Meridional Flow from HMI Using the RingDiagram Pipeline, Sol. Phys., 287, 85-106, 2013

Matsuo, T., Lee, I. \& Anderson, J. L., Thermospheric mass density specification using an ensemble Kalman filter, J. Geophys. Res., 118, 1339-1350, 2013

Miesch, M. S. \& Dikpati, M., A Three-dimensional Babcock-Leighton Solar Dynamo Model ApJ Lett., 785, L8, 1-5, 2014

Muñoz-Jaramillo, A., Nandy, D. \& Martens, P. C. H., The unusual minimum of sunspot cycle 23 caused by meridional plasma flow variations, Nature, $471,80-82,2011$

Sanchez, S., Fournier, A. \& Aubert, J., The Predictability of AdvectionDominated Flux-Transport Dynamo Models, ApJ, 781, 8, 1-15, 2014

Schad, J., Timmer, R. S. \& Roth, H., Global Helioseismic Evidence for a Deeply Penetrating Solar Meridional Flow Consisting of Multiple Flow Cells, ApJ, 778, L38, 1-7, 2013

Ulrich, R. K., Solar Meridional Circulation from Doppler Shifts of the Fe I Line at $5250 \mathrm{~A}^{\circ}$ as Measured by the 150-foot Solar Tower Telescope at the Mt. Wilson Observatory, ApJ, 725, 658-669, 2010

Wang, Y. -M. \& Sheeley, N. R. Jr., Magnetic flux transport and the sun's dipole moment - New twists to the Babcock-Leighton model, ApJ, 375, 761-770, 1991

Yeates, A. R. \& Muñoz-Jaramillo, A., Kinematic active region formation in a three-dimensional solar dynamo model, MNRAS, 436, 3366-3379, 2013

Zhao, J., Bogart, R. S., Kosovichev, A. G., Duvall, T. L., Jr., Hartlep, H., Detection of Equatorward Meridional Flow and Evidence of Double-cell Meridional Circulation inside the Sun, ApJ Lett., 774, L29, 1-6, 2013 